\title{
Comparison between the National Prototype of the kilogram and stainless steel mass standards
}

Adriana Vâlcu*

National Institute of Metrology, Bucharest, Romania

Received: 10 March 2016 / Accepted: 11 April 2016

\begin{abstract}
In 2013, the National Prototype of the Kilogram (NPK) No. 2 belonging to Romania, together with stainless steel kilogram standard $\mathrm{Ni}$ 81, received an updated mass value from BIPM. Therefore, the first task after their receiving from BIPM was to perform comparisons between NPK and reference kilograms stainless steel standards. In the paper are described mass standards, weighing scheme used for comparisons, calculations of mass and associated uncertainties, as the mass change for all weights involved. It is also presented a comparison between Romanian $\mathrm{CMC}(\mathrm{s})$ and other countries for this important stage of dissemination of mass unit.
\end{abstract}

Keywords: National prototype of the kilogram, reference mass standards, weighing scheme, check standard

\section{Introduction}

In 1889, at the First Conference on Weights and Measures (CGPM), the kilogram prototypes were shared randomly - for each country. Romania received the "National Prototype Kilogram No. 2" (NPK 2). Now, it is maintained by the National Institute of Metrology and serves as a reference for the entire dissemination of the mass unit in Romania.

The link between the SI unit of mass and the Romanian national standard of mass is maintained through periodic calibrations of NPK at the BIPM. During its lifetime, the Romanian national standard of mass was calibrated at the BIPM six times: three "periodic verifications" occurred in 1899-1911, 1939-1953, and 1988-1992 and three additionally calibrations in 1976 , 2005 and 2013.

The realization and dissemination of the mass unit by the Mass Laboratory of the Romanian National Institute of Metrology starts from the reference stainless steel standards (a set of three $1 \mathrm{~kg}$ mass standards and two sets of disc weights from $50 \mathrm{~g}$ to $500 \mathrm{~g}$ ), which are traceable to the International Prototype Kilogram through the mass of the Romanian Prototype Kilogram No. 2. This is followed by dissemination to multiples and submultiples of the kilogram covering the range from $0.1 \mathrm{mg}$ to $1000 \mathrm{~kg}$.

In a schematic representation, in Figure 1 is presented this main stage of the Romanian dissemination mass unit from NPK until mass standards of class $\mathrm{E}_{1}$.

The first task after receiving the standards from BIPM was to perform comparisons between NPK and reference stainless steel standards. In the first stage, the reference

^ Correspondence: adivaro@yahoo.com

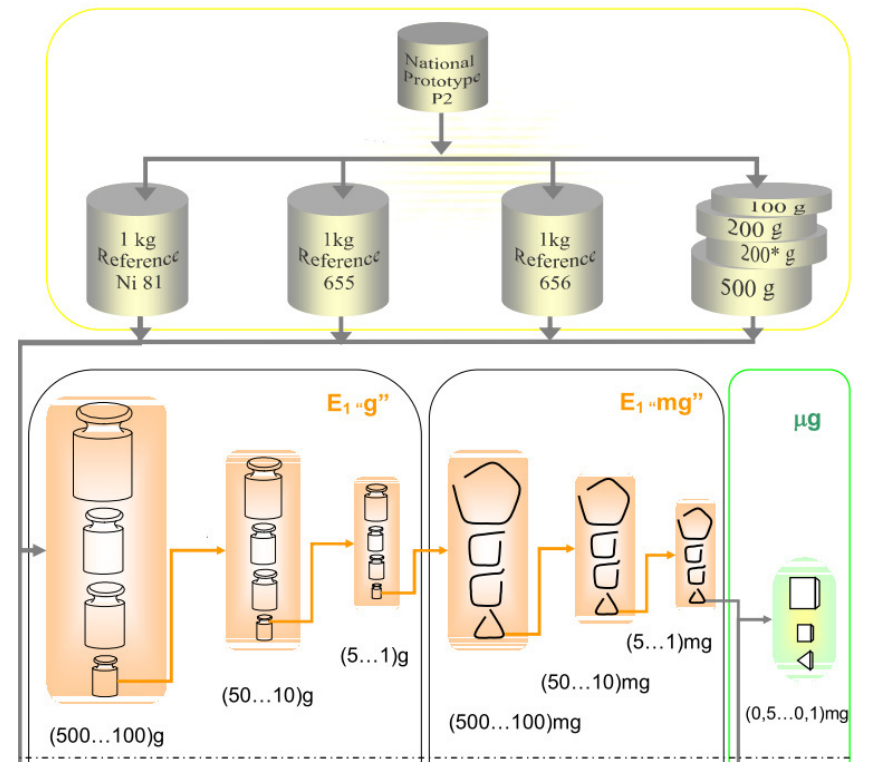

Fig. 1. Schematic representation of the main stage of the dissemination of mass unit.

kilogram standards identified with 655,656 and $\mathrm{Ni} 81$ were compared with NPK and, in a second stage, two sets of disc weights were compared with NPK. For both stages, Ni 81 (considered the most important standard after NPK) acted as check standard. In this paper, the mass comparisons between NPK No. 2 and the three stainless steel kilogram standards are presented. The mass standards, weighing instrument, weighing scheme used for comparisons, calculations of mass and associated uncertainties, as the mass change for all weights involved are described. 
Table 1. The characteristics of the weights involved in the measurements.

\begin{tabular}{cccccc}
\hline Standard ID & Volume $V \mathrm{~cm}^{3}$ & Density, $\rho \mathrm{kg} / \mathrm{m}^{3}$ & $U_{V} \mathrm{~cm}^{3}$ & $U_{\rho} \mathrm{kg} / \mathrm{m}^{3}$ & $\alpha{ }^{\circ} \mathrm{C}^{-1}$ \\
\hline VP2 & 46.4377 & 21534.22 & 0.0012 & 0.56 & $2.6 \times 10^{-5}$ \\
V656 & 124.8407 & 8010.21 & 0.0011 & 0.07 & $4.8 \times 10^{-5}$ \\
VNi & 127.7398 & 7828.41 & 0.0012 & 0.07 & $4.6 \times 10^{-5}$ \\
V655 & 124.8405 & 8010.22 & 0.0011 & 0.07 & $4.8 \times 10^{-5}$ \\
\hline
\end{tabular}

\section{Equipments and standards involved in measurement}

\subsection{Mass comparator}

An automatic mass comparator (AT 1006, manufactured by Mettler-Toledo) was available for the dissemination of mass unit from NPK to stainless steel mass standards. With this automatic mass comparator, batch of weights can be loaded in one step at the beginning and one unloading in the end of the process. The comparator is designed for mass determinations of high accuracy using substitution method. Errors due to operator during the determinations are practically eliminated by automatic operation mode. This comparator gives the possibility to perform measuring cycles ABA, ABBA or AB1 ... BnA, according to reference [1]. The technical specifications for the comparator are:

- Maximum capacity: 1011 g;

- Readability: $1 \mu \mathrm{g}$;

- Repeatability:

$-(2 \ldots 3) \mu \mathrm{g}$ in the determinations with Pt-Ir standard;

- $(0.4 \ldots 1) \mu \mathrm{g}$ in the determinations with stainless steel mass standards.

\subsection{Reference kilograms}

Figure 2 shows the weights involved in the main stage of the dissemination of mass unit:

- NPK is a cylinder with diameter and height of about $39 \mathrm{~mm}$. It is made of an alloy of $90 \%$ Platinum and $10 \%$ Iridium.

- Nicral 81 (Ni 81) was made by "Prolabo" - France in 1978 and received by INM in 1981. It is the most important standard after NPK and it is made of Ni-Cr, having a diameter approximately equal to the height (54 mm).

- The weights denoted with 655 and 656 are made from non-magnetic stainless steel, having a cylindrical form, according to reference [1].

The main characteristics of the weights involved in measurements are listed in Table 1.

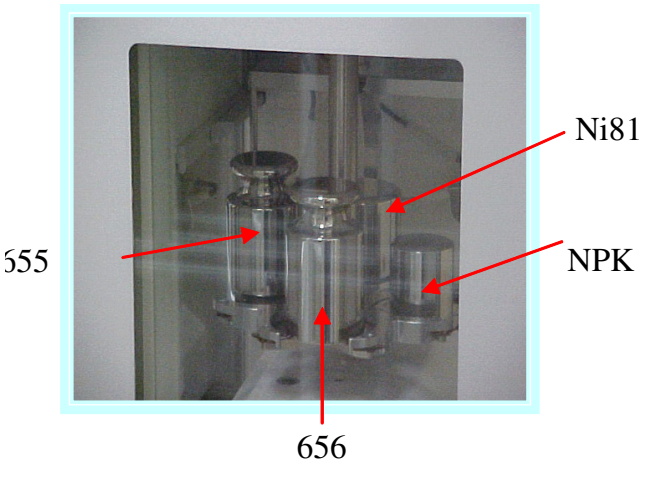

Fig. 2. Kilograms mass standards.

\subsection{Environmental conditions monitoring system}

The mass comparator is equipped with an environmental conditions monitoring system, consisting in a precise "climate station", model Klimet A30.

Apart from this, an instrument for determination of the carbon dioxide concentration from air, type Carbocap GMP343 is used. This instrument is necessary for measuring the $\mathrm{CO}_{2}$ concentration from air, in order to calculate air density and air buoyancy corrections during comparisons performed between the NPK and stainless steel mass standards.

Starting from environmental parameters, the air density is calculated using the formula recommended in reference [2].

\section{Comparison scheme}

The mass standards are compared between them in all possible combinations, as is presented in Figure 3.

In matrix form, the scheme is presented as follows:

$$
X=\left|\begin{array}{cccc}
\mathrm{P}_{2} & \mathrm{Ni} 81 & 656 & 655 \\
1 & -1 & 0 & 0 \\
1 & 0 & -1 & 0 \\
1 & 0 & 0 & -1 \\
0 & 1 & -1 & 0 \\
0 & 1 & 0 & -1 \\
0 & 0 & 1 & -1
\end{array}\right|
$$

In the comparisons, Ni 81 acted as check standard. 


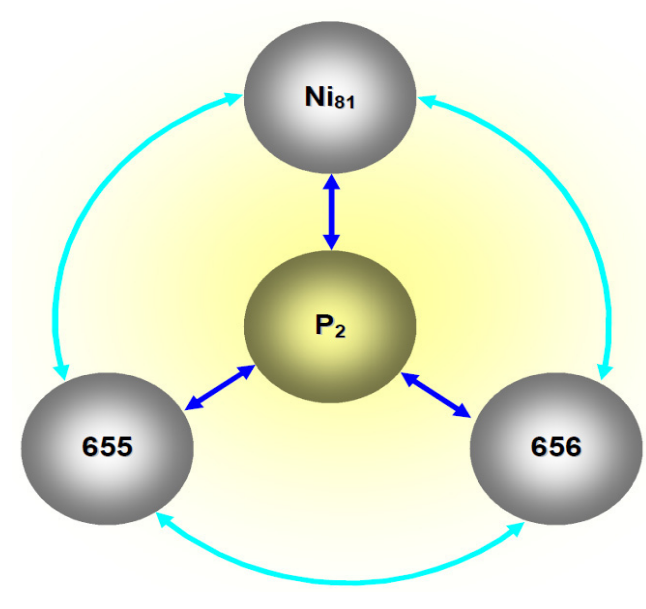

Fig. 3. Comparison scheme.

\section{Processing data obtained in comparisons}

The mathematical model of the comparison can be represented as follow:

$$
Y=X \beta+e
$$

where

$Y(n, 1)$ is the vector of the $n$ observations (including buoyancy corrections);

$\beta(k, 1)$ the vector of the $k$ mass values of the standards; $X(n, k)$ design matrix; entries of the design matrix are $+1,-1$, and 0 according to the role played by each of the parameters (from the vector $\beta$ ) in each comparison;

$e(n, 1)$ vector of the deviations.

Each element of the vector $Y$ is calculated using formula:

$$
y=\Delta m+\rho_{a}\left(V_{j}-V_{r}\right)
$$

$y \quad$ the corrected indication;

$\Delta m$ difference of balance readings calculated from weighing cycles (ABBA);

$\rho_{a} \quad$ air density at the time of the weighing;

$V_{r}, V_{j}$ volumes of the standards involved in a measurement.

If the temperature during the measurements is different than $20^{\circ} \mathrm{C}$, the volume of the weights is corrected using the formula:

$$
V_{t}=V_{20}\left[1+\alpha_{t}(t-20)\right]
$$

where:

$V_{t}$ is the volume of the standard at the working temperature;

$V_{20}$ volume of the standard at $20{ }^{\circ} \mathrm{C}$;

$T$ temperature during comparison;

$\alpha_{t}$ the coefficient of cubic expansion of the material from which the weights are made. Its value depends on the temperature during measurements.
At each difference calculated, is added the correction due to different centre-of-mass levels.

The results of the calibration are obtained with the aid of least squares adjustment which also provides the variance-covariance matrix of the calculated mass values.

The mass of the weights was calculated using the formula [3]:

$$
\langle\beta\rangle=\left(\mathrm{X}^{\prime \mathrm{T}} \mathrm{X}^{\prime}\right)^{-1} \mathrm{X}^{\prime \mathrm{T}} \mathrm{Y}^{\prime}
$$

where:

$$
\mathrm{X}^{\prime}=\mathrm{G} \cdot \mathrm{X} \text { and } \mathrm{Y}^{\prime}=\mathrm{G} \cdot \mathrm{Y}
$$

$G$ is a diagonal matrix containing the diagonal elements:

$$
g_{\mathrm{ii}}=n_{\mathrm{i}} \cdot\left(s_{\mathrm{bal}}^{2} / s_{\mathrm{i}}^{2}\right), \quad i=1 \ldots n
$$

$n_{\mathrm{i}} \quad$ is the ABBA cycles number,

$s_{\text {bal }}$ standard deviation of the balance determined from a complete comparison,

$s_{\mathrm{i}} \quad$ standard deviation of each measurement from a complete comparison,

$X^{\prime \mathrm{T}}$ transpose of $X$ and $\left(X^{\prime \mathrm{T}} \cdot X^{\prime}\right)^{-1}$ is the inverse of $\left(X^{\prime \mathrm{T}} \cdot X^{\prime}\right)$.

\section{Estimating uncertainty}

In evaluating standard uncertainty associated with the results of calibration, the following contributions have to be taken into account:

- type A uncertainty, evaluated by statistical analysis of series of repeated observations;

- type B uncertainty, evaluated by scientific judgment based on all of the available information on the possible variability of an input quantity that has not been obtained from repeated observations, such as:

- reference standard;

- resolution of the weighing instrument;

- sensitivity of the weighing instrument;

- effect of the air buoyancy;

- the difference between centre-of-mass levels of the weights.

The uncertainty components can be graphically represented in an Ishikawa (Fishbone) diagram [4,5], as shown in Figure 4.

\section{Final results obtained from the comparisons}

The results obtained for the three mass standards calibrated are presented in Table 2. 


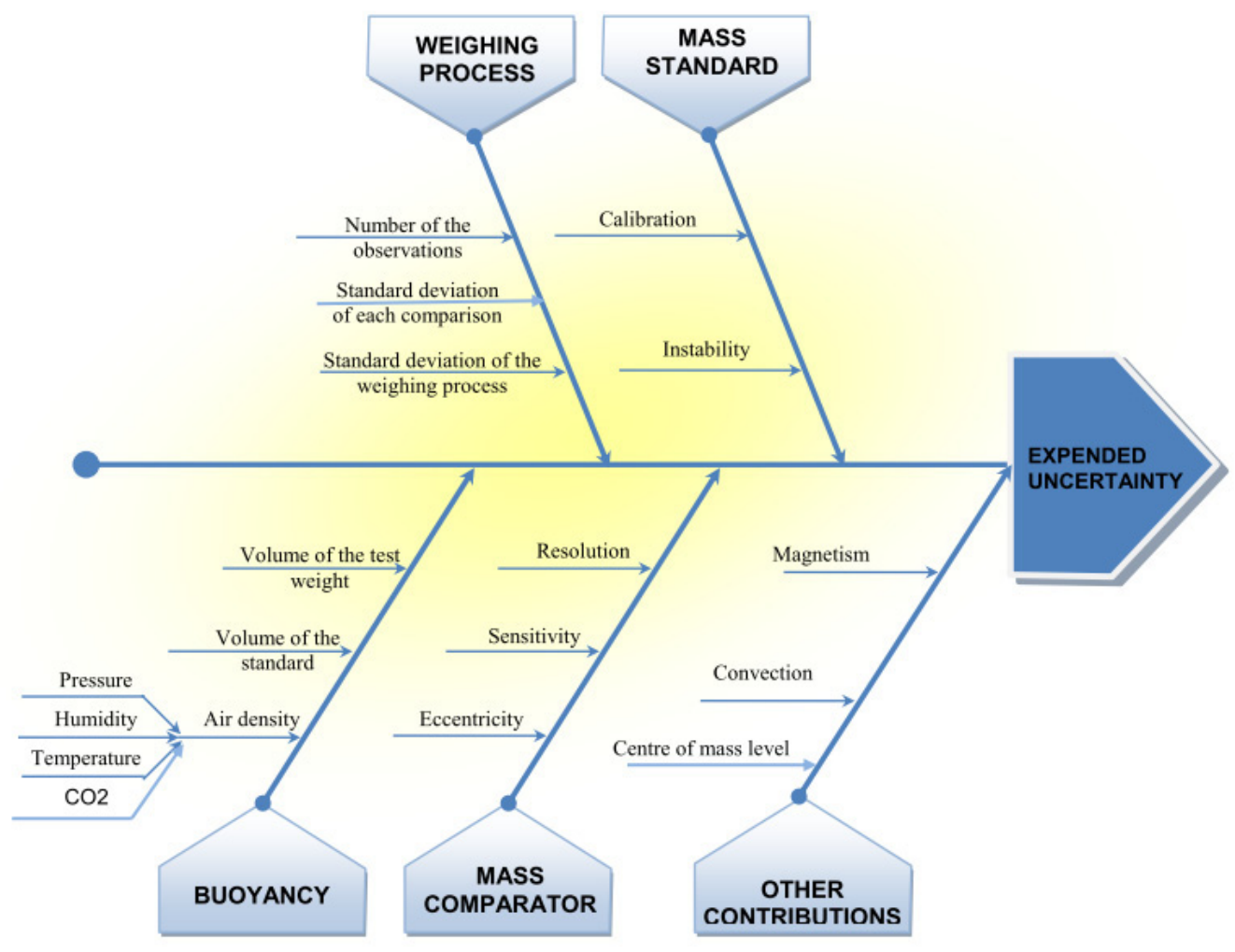

Fig. 4. Ishikawa diagram of uncertainty components.

Table 2. The final results obtained for mass standards.

\begin{tabular}{ccc}
\hline Standard ID & Mass & Uncertainty $U \mathrm{mg}$ \\
\hline Ni 81 & $1 \mathrm{~kg}+0.113 \mathrm{mg}$ & 0.042 \\
655 & $1 \mathrm{~kg}-0.293 \mathrm{mg}$ & 0.042 \\
656 & $1 \mathrm{~kg}-0.203 \mathrm{mg}$ & 0.042 \\
\hline
\end{tabular}

\section{Statistical control regarding quality assessement of the calibration}

The reference weight Ni 81 acted as check standard. To see if its mass value obtained at INM is consistent with previous value received from BIPM, it was calculated the normalized error $E_{n}$, which takes into account the result and uncertainty from the last calibration. In the calibration certificate issued by BIPM is given for $\mathrm{Ni} 81$ the next mass value:

$$
1 \mathrm{~kg}+0.119 \mathrm{mg}, \quad u_{\mathrm{c}}=0.015 \mathrm{mg}(k=1) .
$$

By calculating the normalized error, it can be seen that the consistency of the results is demonstrated.

$$
E_{n}=\frac{\delta_{I N M}-\delta_{B I P M}}{\sqrt{U_{I N M}^{2}+U_{B I P M}^{2}}}=0.12 .
$$

Table 3. Mass change of Ni 81.

\begin{tabular}{cccc}
\hline Data & Mass error mg & Uncertainty $U \mathrm{mg}$ & Drift mg \\
\hline 1981 & 0.123 & 0.023 & \\
Sept. 2005 & 0.130 & 0.014 & 0.007 \\
Sept. 2012 & 0.177 & 0.030 & 0.047 \\
Oct. 2012* & 0.123 & 0.030 & -0.056 \\
Nov. 2012 & 0.120 & 0.030 & -0.003 \\
May 2013 & 0.119 & 0.030 & -0.001 \\
\hline
\end{tabular}

${ }^{*}$ The standard was cleaned.

\section{Mass change of stainless steel standards}

\subsection{Kilogram Ni 81}

Analysis of the behaviour in time for the standard $\mathrm{Ni} 81$, presented in Table 3 and Figure 5, was performed using only the mass values obtained at BIPM. One can observe a good stability of this standard. It is noted the fact that, the mass value obtained in October 2012 was given after the cleaning of the standard.

\subsection{Kilogram 655}

Analysis of the behaviour in time for the standard 655 is presented in Table 4 and Figure 6. Excepting the value from 2010, when the density of the weight was determined, it can be seen o good stability in time of this mass standard. 
Adriana Vâlcu: Comparison between the National Prototype of the kilogram and stainless steel mass standards 205-p5

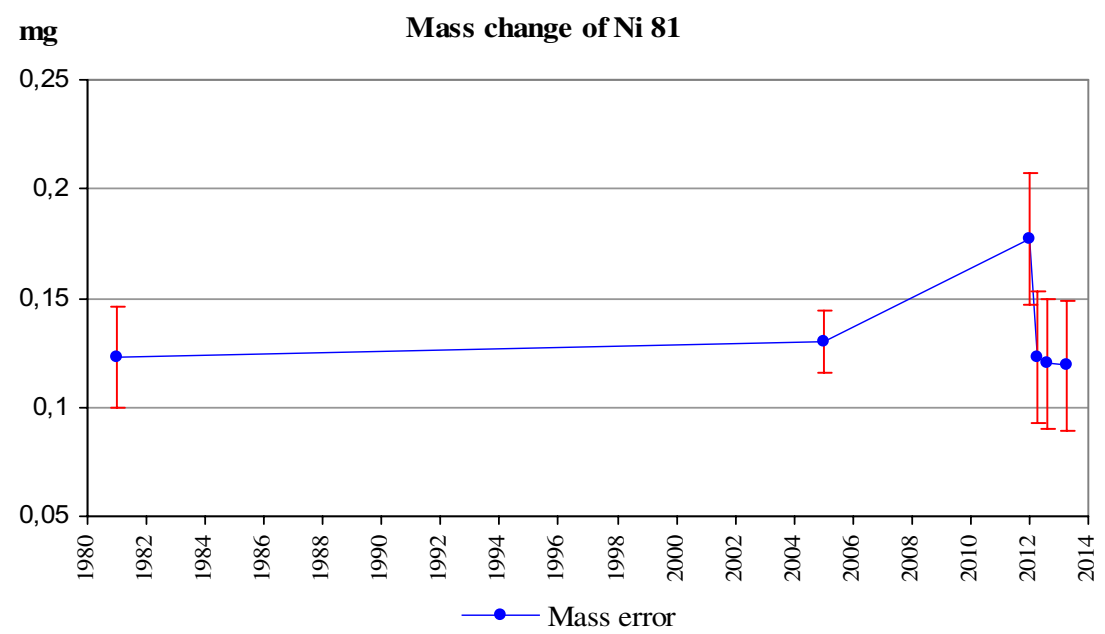

Fig. 5. Mass change of Ni 81 between 1981-2013.

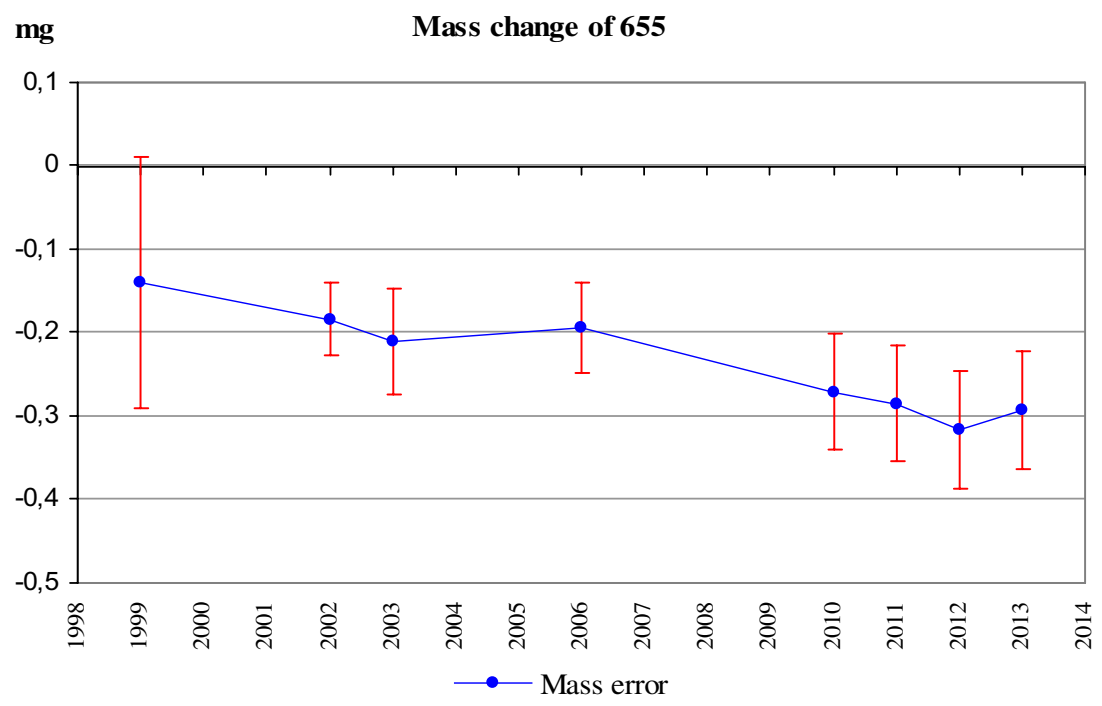

Fig. 6. Mass change of 655 between 1999-2013.

Table 4. Mass change of 655 .

\begin{tabular}{cccc}
\hline Data & Mass error mg & Uncertainty $U \mathrm{mg}$ & Drift mg \\
\hline Mar.1999 & -0.14 & 0.15 & \\
Dec.2002 & -0.184 & 0.043 & -0.044 \\
Nov.2003 & -0.211 & 0.064 & -0.027 \\
Dec.2006 & -0.194 & 0.054 & 0.017 \\
Ian.2010* & -0.271 & 0.070 & -0.077 \\
Feb.2011 & -0.285 & 0.070 & -0.014 \\
July 2012 & -0.316 & 0.070 & -0.031 \\
Nov. 2013 & -0.291 & 0.070 & +0.025 \\
\hline
\end{tabular}

* It was determined the density of the weight.

\subsection{Kilogram 656}

In Table 5 and Figure 7 is presented analysis of the behaviour in time for the standard 656. After determination
Table 5. Mass change of 656 .

\begin{tabular}{cccc}
\hline Data & Mass error mg & Uncertainty $U \mathrm{mg}$ & Drift $\mathrm{mg}$ \\
\hline Mar.1999 & -0.19 & 0.15 & \\
Dec.2002 & -0.187 & 0.048 & 0.003 \\
Nov.2003 & -0.257 & 0.046 & -0.070 \\
Dec.2006 & -0.142 & 0.026 & 0.115 \\
Ian.2010* & -0.224 & 0.070 & -0.082 \\
Feb.2011 & -0.250 & 0.070 & -0.026 \\
Feb. 2012 & -0.241 & 0.070 & +0.009 \\
Nov. 2013 & -0.200 & 0.070 & +0.041 \\
\hline
\end{tabular}

* It was determined the density of the weight.

of density in 2010 it can be seen a satisfactory stability of the standard 656 .

However, laboratory considered that for the next period the standards will be recalibrated at an interval of 2 years. 


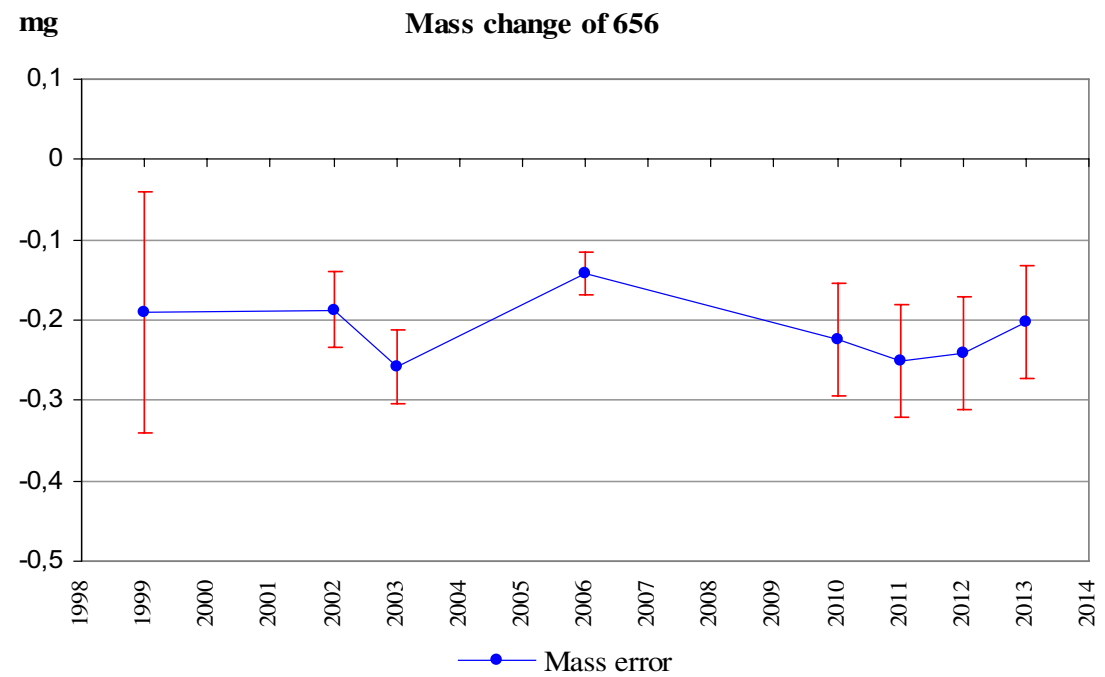

Fig. 7. Mass change of 656 between 1999-2013.

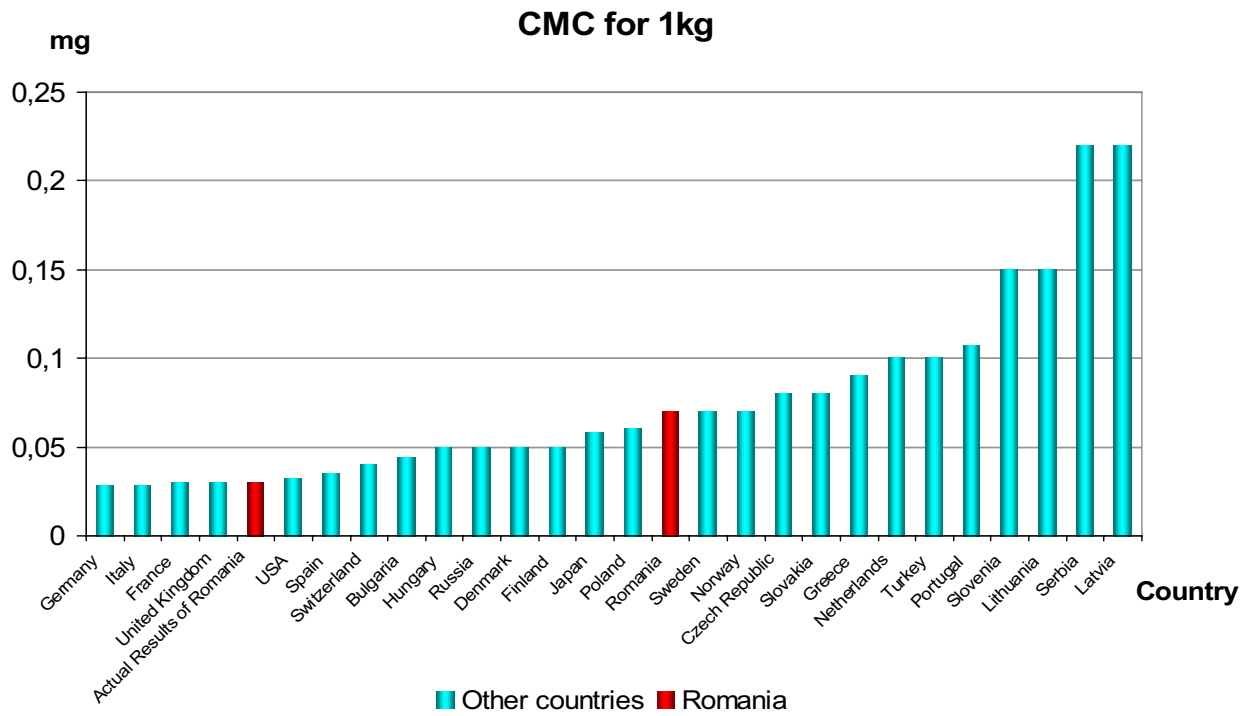

Fig. 8. Comparison between $\mathrm{CMC}(\mathrm{s})$ at $1 \mathrm{~kg}$ level.

\section{Comparison between $\mathrm{CMC}(\mathrm{S})$ at $1 \mathrm{~kg}$ level}

In Figure 8 is presented a comparison between Romanian CMC(s) and other countries for this important stage of dissemination of mass unit. The graph shows the current stage of our CMC and the new position of Romania if the results from Table 2 would be confirmed.

\section{Conclusions}

The paper presents the comparison between the Romanian National Prototype of the Kilogram and three stainless steel mass standards marked as: Ni 81, 655 and 656. In the comparisons, the mass standard Ni 81 acted as check standard. The analyzed results using the normalized error showed a good consistency.

From the performed analysis regarding the mass change for the standards 655 and 656 , it was decided that they will be recalibrated at 2 years.
Although for expanded uncertainty were obtained the values from Table 2 , in the calibration certificate was recorded the value covered by Romanian CMC, namely, $U=0.07 \mathrm{mg}$.

\section{References}

1. OIML, International Recommendation No. 111, Weights of classes E1. E2. F1. F2. M1. M2. M3 (2004), pp. 5-71.

2. A. Picard, R. Davis, M. Glaser, K. Fujii, Revised formula for the density of moist air, CIPM (2007).

3. R. Schwartz, Guide to mass determination with high accuracy, PTB MA-40, 3 and 54-58 (1995).

4. A. Vâlcu, Extension of dissemination of mass unit below $1 \mathrm{mg}$ in Romania, Metrologie Revue LX, 21-28 (2014)

5. A. Vâlcu, Extension of dissemination of mass unit below $1 \mathrm{mg}$ in Romania, Proceedings of International Metrology Congress, Paris, 2013 